arXiv version: fonts, pagination and layout may vary from GTM published version

\title{
Open string instantons and relative stable morphisms
}

\author{
JUN LI \\ YUN S SONG
}

\begin{abstract}
We show how topological open string theory amplitudes can be computed by using relative stable morphisms in the algebraic category. We achieve our goal by explicitly working through an example which has been previously considered by Ooguri and Vafa from the point of view of physics. By using the method of virtual localization, we successfully reproduce their results for multiple covers of a holomorphic disc, whose boundary lies in a Lagrangian submanifold of a Calabi-Yau 3-fold, by Riemann surfaces with arbitrary genera and number of boundary components. In particular we show that in the case we consider there are no open string instantons with more than one boundary component ending on the Lagrangian submanifold.
\end{abstract}

14N35; 14D21

Reproduced by kind permission of International Press from:

Advances in Theoretical and Mathematical Physics, Volume 5 (2002) pages 69-91

\section{Introduction}

The astonishing link between intersection theories on moduli spaces and topological closed string theories has by now taken a well-established form, a progress for which $\mathrm{E}$ Witten first plowed the ground in his seminal paper [21]. As a consequence, there now exist rigorous mathematical theories of Gromov-Witten invariants, which naturally arise in the aforementioned link. In the symplectic category, Gromov-Witten invariants were first constructed for semi-positive symplectic manifolds by Y Ruan and G Tian [18]. To define the invariants in the algebraic category, J Li and G Tian constructed the virtual fundamental class of the moduli space of stable maps by endowing the moduli space with an extra structure called a perfect tangent-obstruction complex [15]. ${ }^{1}$ Furthermore, Gromov-Witten theory was later extended to general symplectic manifolds by Fukaya and Ono [3], and by Li and Tian [14]. In contrast to such an impressive list of advances

\footnotetext{
${ }^{1}$ Alternative constructions were also made by Y Ruan [17] and by B Siebert [19].
} 
just described, no clear link currently exists between topological open string theories and intersection theories on moduli spaces. One of the most formidable obstacles that stand in the way to progress is that it is not yet known how to construct well-defined moduli spaces of maps between manifolds with boundaries. The main goal of this paper is to contribute to narrowing the existing gap between topological open string theory and Gromov-Witten theory. In so doing we hope that our work will serve as a stepping-stone that will take us a bit closer to answering how relative stable morphisms can be used to study topological open string theory.

In order to demonstrate the proposed link between topological open string theory and Gromov-Witten theory, we will focus on an explicit example throughout the paper. The same example was also considered by string theorists Ooguri and Vafa in [16], where they used results from Chern-Simons theory and M-theory to give two independent derivations of open string instanton amplitudes. A more detailed description of the problem will be presented later in the paper. We just mention here that, by using our mathematical approach, we have successfully reproduced their answers for multiple covers of a holomorphic disc by Riemann surfaces of arbitrary genera and number of holes. In fact we show that there are no open string instantons with more than one hole, a result which was anticipated in [16] from their physical arguments.

The invariants we compute are a generalization of absolute Gromov-Witten invariants that should be more familiar to string theorists. Our case involves relative stable maps which intersect a specified complex-codimension-two submanifold of the target space in a finite set of points with multiplicity. It will become clear later in the paper that the theory of relative stable maps is tailor-made for studying topological open string theory. The construction of relative stable maps was first developed in the symplectic category (Li-Ruan [11], Ionel-Parker [6, 7]). Recently in [13, 12] the first author of the present paper has given an algebro-geometric definition of the moduli space of relative stable morphisms and has constructed relative Gromov-Witten invariants in the algebraic category. The foundation of our work will be based on those papers.

The organization of this paper is as follows: In Section 1 we give a brief description of the multiple cover problem that arose in [16] and state what we wish to reproduce using relative stable morphisms. In Section 2 we define the moduli space of relative stable morphisms and describe how multiple covers of a holomorphic disc can be viewed as a problem regarding relative stable morphisms. We investigate the obstruction theory of the moduli space in Section 3. In Section 4 we study the localization of the virtual fundamental class and compute the equivariant Euler class of the virtual normal bundle 
to the fixed locus. In Section 5 we evaluate the relevant invariants for the case where the source Riemann surface has only one boundary component. The cases with more than one boundary component are subsequently discussed in Section 6. We conclude in Section 7 with some comments.

Note While this manuscript was in its final stage of preparation, Katz and Liu announced their results [9] which deal with the same subject matter as our paper.

\section{A brief description of the problem}

The notion of duality has been one of the most important common threads that run through modern physics. A duality draws intricate connections between two seemingly unrelated theories and often allows one to learn about one theory from studying the other. A very intriguing duality correspondence has been proposed by Gopakumar and Vafain [4], where the authors provide several supporting arguments for a duality between the large- $N$ expansion of $S U(N)$ Chern-Simons theory on $S^{3}$ and a topological closed string theory on the total space of the vector bundle $\mathcal{O}_{\mathbf{P}^{1}}(-1) \oplus \mathcal{O}_{\mathbf{P}^{1}}(-1)$ over $\mathbf{P}^{1} .^{2}$ The equivalence was established in [4] at the level of partition functions. We know from Witten's work in [22], however, that there are Wilson loop observables in Chern-Simons theory which correspond to knot invariants. The question then is, "What do those invariants that arise in Chern-Simons theory correspond to on the topological string theory side?"

The first explicit answer to the above question was given by Ooguri and Vafa in [16]. In the case of a simple knot on $S^{3}$, by following through the proposed duality in close detail, they showed that the corresponding quantities on the topological string theory side are open string instanton amplitudes. More precisely, in the particular example they consider, the open string instantons map to either the upper or the lower hemisphere of the base $\mathbf{P}^{1}$. $^{3}$

\footnotetext{
${ }^{2}$ See [4] and references therein for a more precise account of the proposal.

${ }^{3} \mathrm{We}$ clarify that the geometric set up in the present case is no longer that described above. There is a unique Lagrangian 3-cycle $\mathcal{C}_{K}$ in $T^{*} S^{3}$ which intersects $S^{3}$ along a given knot $K$ in $S^{3}$. Associated to such a 3 -cycle $\mathcal{C}_{K}$ in $T^{*} S^{3}$ there is a Lagrangian 3-cycle $\tilde{\mathcal{C}_{K}}$ in the local Calabi-Yau three-fold $X$ of the topological string theory side. For the simple knot $S$ considered by Ooguri and Vafa, the latter 3 -cycle $\tilde{\mathcal{C}}_{S}$ intersects the base $\mathbf{P}^{1}$ of $X$ along its equator. It is the presence of this 3-cycle that allows for the existence of holomorphic maps from Riemann surfaces with boundaries to either the upper or the lower hemisphere. See [16] for a more detailed discussion.
} 
According to [16], the generating function for topological open string amplitudes is

$$
F(t, V)=\sum_{g=0}^{\infty} \sum_{h=0}^{\infty} \sum_{d_{1}, \ldots, d_{h}}^{\infty} \lambda^{2 g-2+h} F_{g ; d_{1}, \ldots, d_{h}}(t) \prod_{i=1}^{h} \operatorname{tr} V^{d_{i}},
$$

where $t$ is the Kähler modulus of $\mathbf{P}^{1} ; V$ is a path-ordered exponential of the gauge connection along the equator and $\operatorname{tr} V^{d_{i}}$ arises from the $i^{\text {th }}$ boundary component which winds around the equator $\left|d_{i}\right|$-times with orientation, which determines the sign of $d_{i}$; $\lambda$ is the string coupling constant; and $F_{g ; d_{1}, \ldots, d_{h}}$ is the topological open string amplitude on a genus $-g$ Riemann surface with $h$ boundary components. Furthermore, by utilizing the aforementioned duality with Chern-Simons theory, Ooguri and Vafa concluded that

$$
F(t, V)=i \sum_{d=1}^{\infty} \frac{\operatorname{tr} V^{d}+\operatorname{tr} V^{-d}}{2 d \sin (d \lambda / 2)} e^{-d t / 2},
$$

which they confirmed by using an alternative approach in the M-theory limit of type IIA string theory. ${ }^{4}$ By comparing $1-1$ and $1-2$, one immediately sees that there are no open string instantons with more than one boundary component ending on the equator; that is, $F_{g ; d_{1}, \ldots, d_{h}}=0$ for $h>1$. To extract the topological open string amplitude on a genus $-g$ Riemann surface with one boundary component $(h=1)$, we need to expand $1-2$ in powers of $\lambda$. After some algebraic manipulation, we see that

$$
\begin{array}{r}
F(t, V)=i \sum_{d=1}^{\infty}\left(\frac{1}{d^{2}} \lambda^{-1}+\sum_{g=1}^{\infty} d^{2 g-2} \frac{2^{2 g-1}-1}{2^{2 g-1}} \frac{\left|B_{2 g}\right|}{(2 g) !} \lambda^{2 g-1}\right) \\
e^{-d t / 2}\left(\operatorname{tr} V^{d}+\operatorname{tr} V^{-d}\right),
\end{array}
$$

where $B_{2 g}$ are the Bernoulli numbers defined by

$$
\sum_{n=0}^{\infty} B_{n} \frac{x^{n}}{n !}=\frac{x}{e^{x}-1} .
$$

Hence, topological open string amplitudes, which correspond to multiple covers of either the upper or the lower hemisphere inside the local Calabi-Yau three-fold described above, are

$$
-i F_{g ; d_{1}, \ldots, d_{h}}(0)= \begin{cases}d^{-2}, & g=0, h=1,\left|d_{1}\right|=d>0, \\ d^{2 g-2}\left(\frac{2^{2 g-1}-1}{2^{2 g-1}} \frac{\left|B_{2 g}\right|}{(2 g) !}\right), & g>0, h=1,\left|d_{1}\right|=d>0, \\ 0, & \text { otherwise. }\end{cases}
$$

\footnotetext{
${ }^{4}$ We refer the reader to the original reference [16] for further description of this approach.
} 
In the remainder of this paper, we will work towards reproducing these results using relative stable morphisms.

\section{Relative stable maps and Lagrangian submanifolds}

In this section, we will formulate the problem in terms of the moduli space of stable holomorphic maps. Throughout this paper, we fix two points $q_{0}$ and $q_{\infty} \in \mathbf{P}^{1}$, and choose a homogeneous coordinate $\left[w_{1}, w_{2}\right]$ of $\mathbf{P}^{1}$ so that $q_{0}\left(\right.$ resp. $\left.q_{\infty}\right)$ is the point $[0,1]$ (resp. [1,0]). We will use $w$ to denote the standard coordinate of $\mathbf{C}=\mathbf{P}^{1}-q_{\infty}$ so that $w$ is related to the homogeneous coordinate $\left[w_{0}, w_{1}\right]$ via $w=w_{0} / w_{1}$. Hence $q_{0}$ and $q_{\infty}$ become 0 and $\infty$, respectively. We let $W$ be the total space of the vector bundle $\mathbf{1}_{\mathbb{C}} \oplus \mathbf{1}_{\mathbb{C}}$ over $\mathbf{C}=\mathbf{P}^{1}-q_{\infty}$. (In this paper, we will use $\mathbf{1}_{X}$ to denote the trivial holomorphic line bundle over $X$.) For any $r \in \mathbb{R}^{+}$we denote by $D_{r} \subset \mathbf{C}$ the closed disk $|w| \leq r^{d}$ and denote the boundary of $D_{r}$ by $\partial D_{r}$. Furthermore, we let $W_{r}=\pi^{-1}\left(D_{r}\right)$, where $\pi: W \rightarrow \mathbf{C}$ is the projection. In the present section, we will consider the space of holomorphic maps from Riemann surfaces to $W_{r}$ whose boundary lies in a Lagrangian submanifold in $\partial W_{r}$.

We now describe this Lagrangian submanifold, following Ooguri and Vafa [16]. We let $e_{1}$ and $e_{2}$ be the constant 1 section of the first and the second factor of $\mathbf{1}_{\mathbb{C}} \oplus \mathbf{1}_{\mathbb{C}}$. Then any vector $\xi \in \mathbf{1}_{\mathbb{C}} \oplus \mathbf{1}_{\mathbb{C}}$ over $s \in \mathbf{C}$ is expressed uniquely as $\xi=\left(s, u e_{1}+v e_{2}\right)$. In case $w$ is the coordinate of $s \in \mathbf{C}$, we say $(w, u, v)$ is the coordinate of $\xi \in W$. The Lagrangian submanifold introduced by Ooguri and Vafa in [16] is ${ }^{5}$

$$
B_{r}=\left\{(w, u, v)|| w \mid=r^{d}, u=\bar{w} \bar{v}\right\} .
$$

As a convention in this paper, we will use $t=e^{i \theta}$ to denote a general element in the group $S^{1}$. We fix an $S^{1}$-action on $\mathbf{C} \subset \mathbf{P}^{1}$ via

$$
[w, 1]^{t}=[t w, 1] .
$$

Then $w^{t}=t^{-1} w$, where $w^{t}$ is the push-forward of the function $w$ under the group action, which is the pull-back of $w$ under the inverse of 2-2. We next choose an $S^{1}$-linearization of $W \rightarrow \mathbf{C}$ so that it leaves $B_{r}$ invariant. We choose

$$
e_{1}^{t}=t e_{1} \quad \text { and } \quad e_{2}^{t}=e_{2} .
$$

\footnotetext{
${ }^{5}$ The authors of [16] considered the Lagrangian submanifold $u_{-}=w \bar{v}_{-}$, where $u_{-}$and $v_{-}$ are given as sections of $\mathcal{O}_{\mathbf{P}^{1}}(-1)$ using a frame over $\mathbf{P}^{1}-q_{0}$. The transition between $u$ and $u_{-}$ is $u_{-}=w u$. Under this condition, our equation is equivalent to that of Ooguri and Vafa.
} 
Then $\left(s, u e_{1}+v e_{2}\right)^{t}=\left(s^{t}, u e_{1}^{t}+v e_{2}^{t}\right)$, or equivalently

$$
(w, u, v)^{t}=\left(t^{-1} w, t u, v\right) .
$$

Hence $(u-\bar{w} \bar{v})^{t}=t u-t \bar{w} \bar{v}$ and thus $B_{r}$ is $S^{1}$-invariant.

We now define the moduli space $\mathbf{M}_{g, \mu}^{\text {rel }}\left(W_{r}, B_{r}\right)$ of relative holomorphic maps from genus- $g$ Riemann surfaces with boundaries to $\left(W_{r}, B_{r}\right)$, with prescribed winding numbers along the boundaries of the domain Riemann surfaces. We let $g \geq 0$ be an integer, and $\mu=\left(d_{1}, \cdots, d_{n}\right)$ an $n$-tuple of positive integers. In this paper, we will call connected, holomorphic nodal curves with ordered smooth boundaries prestable nodal holomorphic curves with ordered boundaries. When $\Sigma$ is such a curve we will use $\partial_{k} \Sigma$ to denote the $k^{\text {th }}$ boundary component of $\Sigma$. Naturally, we give each boundary $\partial_{k} \Sigma$ its induced orientation. Now let $\Sigma$ be a holomorphic nodal curve with $n$ ordered boundary components and let $f:(\Sigma, \partial \Sigma) \rightarrow\left(W, B_{r}\right)$ be a holomorphic map. We say $f$ has winding number $d_{k}$ along the $k^{\text {th }}$ boundary component if the degree of

$$
\left.\pi \circ f\right|_{\partial_{k} \Sigma}: \partial_{k} \Sigma \rightarrow \partial D_{r}
$$

has degree $d_{k}$. We say $f$ is a relative holomorphic map (with $\mu=\left(d_{1}, \cdots, d_{n}\right.$ ) implicitly understood) if, for all $k$, it has winding number $d_{k}$ along its $k^{\text {th }}$ boundary component. A relative holomorphic map is said to be stable if there is no irreducible component of $\Sigma$ that is isomorphic to $\mathbf{P}^{1}$ that contains only one nodal point and that is mapped to a single point in $W_{r}$. As usual, two such relative stable maps $\left(f_{1}, \Sigma_{1}\right)$ and $\left(f_{2}, \Sigma_{2}\right)$ are equivalent if there is an isomorphism $\rho: \Sigma_{1} \rightarrow \Sigma_{2}$ that preserves the order of the boundary components so that $f_{1}=\rho \circ f_{2}$. We define $\mathbf{M}_{g, \mu}^{\mathrm{rel}}\left(W, B_{r}\right)$ to be the moduli space of all relative stable maps from genus- $g$ curves to $\left(W_{r}, B_{r}\right)$, modulo equivalence. Similarly, we define $\mathbf{M}_{g, \mu}^{\mathrm{rel}}\left(D_{r}\right)$ to be the moduli space of all relative stable maps from genus $-g$ curves with ordered boundaries to $\left(D_{r}, \partial D_{r}\right)$, modulo equivalence relation. Since $\left(D_{r}, \partial D_{t}\right) \subset\left(W_{r}, B_{r}\right), \mathbf{M}_{g, \mu}^{\mathrm{rel}}\left(D_{r}\right)$ is a subspace of $\mathbf{M}_{g, \mu}^{\mathrm{rel}}\left(W_{r}, B_{r}\right)$.

We now introduce another moduli space, the moduli space of relative stable morphisms of genus $-g$ to $\left(\mathbf{P}^{1}, q_{\infty}\right)$, with prescribed ramification orders. Let $g$ and $\mu$ be as above. An ordinary relative stable morphism of genus $-g$ and of ramification order $\mu$ consists of a connected $n$-pointed nodal algebraic curve $\left(C, x_{1}, \cdots, x_{n}\right)$ and a morphism $f: C \rightarrow \mathbf{P}^{1}$, so that

$$
f^{-1}\left(q_{\infty}\right)=d_{1} x_{1}+\cdots+d_{n} x_{n}
$$

as a divisor. We say $(f, C)$ (with $\mu$ understood implicitly) is a relative stable morphism if $f$ is stable as a morphism from $C$ to $\mathbf{P}^{1}$. We let $\mathbf{M}_{g, \mu}^{\text {rel }}\left(\mathbf{P}^{1}\right)_{0}$ be the moduli space of 
ordinary relative stable morphisms, with ramification order $\mu$, from genus $-g$ algebraic curves to $\mathbf{P}^{1}$. It is a Deligne-Mumford stack. In [13], the first author of the present paper has constructed the moduli space $\mathbf{M}_{g, \mu}^{\text {rel }}\left(\mathbf{P}^{1}\right)$ of relative stable morphisms, with ramification order $\mu$, from genus- $g$ algebraic curves to $\mathbf{P}^{1}$. The space $\mathbf{M}_{g, \mu}^{\mathrm{rel}}\left(\mathbf{P}^{1}\right)$ is a proper Deligne-Mumford stack. It admits a natural perfect obstruction theory and hence admits a virtual moduli cycle. The moduli stack $\mathbf{M}_{g, \mu}^{\mathrm{rel}}\left(\mathbf{P}^{1}\right)$ contains $\mathbf{M}_{g, \mu}^{\mathrm{rel}}\left(\mathbf{P}^{1}\right)_{0}$ as its open substack. The new ingredient of this moduli space is that a relative stable morphism $f \in \mathbf{M}_{g, \mu}^{\mathrm{rel}}\left(\mathbf{P}^{1}\right)$ consists of an $n$-pointed genus- $g$ algebraic curves $\left(C, x_{1}, \cdots, x_{n}\right)$ and a morphism $f: C \rightarrow \mathbf{P}^{1}[m]$, so that

$$
f^{-1}\left(q_{\infty}\right)=d_{1} x_{1}+\cdots+d_{n} x_{n} .
$$

Here $\mathbf{P}^{1}[m]$ is the nodal curve that has $m$ ordered irreducible components, each isomorphic to $\mathbf{P}^{1}$, so that the $j^{\text {th }}$ component intersects with the $(j+1)^{\text {th }}$ component of $\mathbf{P}^{1}[m]$ at exactly one point. $\mathbf{P}^{1}[m]$ also contains a distinguished divisor $q_{\infty}$ in the first component of $\mathbf{P}^{1}[m]$, and it comes with a projection $\varphi: \mathbf{P}^{1}[m] \rightarrow \mathbf{P}^{1}$ that maps $q_{\infty} \in \mathbf{P}^{1}[m]$ to $q_{\infty} \in \mathbf{P}^{1}$. Moreover, the restriction of $\varphi$ to the last component is an isomorphism to $\mathbf{P}^{1}$, and $\varphi$ contracts all other components of $\mathbf{P}^{1}[m]$. For any morphism $f: C \rightarrow \mathbf{P}^{1}[m]$ we define $\operatorname{Aut}(f)^{\text {rel }}$ to be the set of pairs $(a, b)$, where $a: C \rightarrow C$ are automorphisms of $\left(C, x_{1}, \cdots, x_{n}\right)$ and $b: \mathbf{P}^{1}[m] \rightarrow \mathbf{P}^{1}[m]$ are automorphisms ${ }^{6}$ so that $f \circ a=b \circ f$. We say $f$ is stable if $\operatorname{Aut}(f)^{\mathrm{rel}}$ is finite. From this description, we see that $\mathbf{M}_{g, \mu}^{\mathrm{rel}}\left(\mathbf{P}^{1}\right)_{0} \subset \mathbf{M}_{g, \mu}^{\mathrm{rel}}\left(\mathbf{P}^{1}\right)$ is the open subset consisting of relative stable morphisms $f$ whose codomain is $\mathbf{P}^{1}[1]=\mathbf{P}^{1}$.

We now investigate the moduli space $\mathbf{M}_{g, \mu}^{\text {rel }}\left(W_{r}, B_{r}\right)$ of relative stable holomorphic maps. Let $(f, \Sigma) \in \mathbf{M}_{g, \mu}^{\mathrm{rel}}\left(W_{r}, B_{r}\right)$ be any relative stable map. Composed with $\pi: W_{r} \rightarrow B_{r}$, we obtain a new map

$$
\tilde{f}=\pi \circ f: \Sigma \rightarrow D_{r}
$$

Since fibers of $W_{r} \rightarrow D_{r}$ are vector spaces, $(\tilde{f}, \Sigma)$ is also relative stable and hence is in $\mathbf{M}_{g, \mu}^{\mathrm{rel}}\left(D_{r}\right)$. This defines a map

$$
\mathbf{M}_{g, \mu}^{\mathrm{rel}}\left(W_{r}, B_{r}\right) \longrightarrow \mathbf{M}_{g, \mu}^{\mathrm{rel}}\left(D_{r}\right)
$$

We now show that this is an isomorphism. Given $f \in \mathbf{M}_{g, \mu}^{\mathrm{rel}}\left(W_{r}, B_{r}\right)$ and its induced $\tilde{f} \in \mathbf{M}_{g, \mu}^{\mathrm{rel}}\left(D_{r}\right)$, the original $f$ is given by a holomorphic section

$$
s \in \Gamma\left(\Sigma, \tilde{f}^{*} L_{1} \oplus \tilde{f}^{*} L_{2}\right)
$$

\footnotetext{
${ }^{6} \mathrm{By}$ this we mean that $b$ is an isomorphism of $\mathbf{P}^{1}[m]$ that preserves $q_{\infty} \in \mathbf{P}^{1}[m]$ and the projection $\varphi: \mathbf{P}^{1}[m] \rightarrow \mathbf{P}^{1}$.
} 
We now show that $s=0$. We let $\Sigma_{2}$ be the nodal curve $\Sigma$ with the conjugate holomorphic structure. Namely, $\Sigma_{2}$ is diffeomorphic to $\Sigma$, its conformal structure on each irreducible components is identical to that of $\Sigma$, and its orientation is the opposite of that of $\Sigma$. We let $\Sigma_{1}$ be the identical copy of $\Sigma$. We then let $\tilde{\Sigma}$ be the nodal holomorphic curve derived by identifying the boundary of $\Sigma_{1}$ with the boundary of $\Sigma_{2}$ using the identity map between their boundaries. By the reflection principle, the complex structures of $\Sigma_{1}$ and $\Sigma_{2} \subset \tilde{\Sigma}$ extend to a holomorphic structure on $\tilde{\Sigma}$. The new curve $\tilde{\Sigma}$ is a nodal curve without boundary. We next introduce a line bundle $\tilde{L}$ on $\tilde{\Sigma}$. We pick a sufficiently small open neighborhood $V_{i} \subset \tilde{\Sigma}$ of $\Sigma_{i} \subset \tilde{\Sigma}$. We let $\left.\tilde{L}\right|_{V_{i}} \cong \mathcal{O}_{V_{i}}$ and let $\zeta_{i}$ be the section 1 in $\mathcal{O}_{V_{i}}$. The identification of $\left.\tilde{L}\right|_{V_{1}}$ with $\left.\tilde{L}\right|_{V_{2}}$ over $V_{1} \cap V_{2}$ is given by

$$
\zeta_{1}=f^{*}(w) r^{-2 d} \zeta_{2}
$$

Now let $s$ in $2-4$ be given by $s(z)=u(z) e_{1}+v(z) e_{2}$. Then $u(z) \zeta_{1}$ is naturally a holomorphic section of $\left.\tilde{L}\right|_{\Sigma_{1}}$ and $\bar{v}(z) \zeta_{2}$ also is a holomorphic section of $\left.\tilde{L}\right|_{\Sigma_{2}}$. The condition that $f(z) \in B_{r}$ for $z \in \partial \Sigma$ implies that $u(z)=\overline{f(z) \overline{v(z)}} z \in \partial \Sigma$. Since $|f(z)|=r^{d}$, we have

$$
u(z) \zeta_{1}=\overline{f(z)} \overline{v(z)} f(z) r^{-2 d} \zeta_{2}=\overline{v(z)} \zeta_{2} .
$$

This implies that $u \zeta_{1}$ and $\bar{v} \zeta_{2}$ patch together to form a continuous section of $\tilde{L}$. Since $u \zeta_{1}$ and $\bar{v} \zeta_{2}$ are holomorphic over $\Sigma_{1}$ and $\Sigma_{2}$, respectively, $\tilde{s}$ is holomorphic. Finally, it is straightforward to check that $\operatorname{deg} \tilde{L}=-\sum d_{k}<0$. Hence $\tilde{s} \equiv 0$, which shows that $f \equiv \tilde{f}$. This proves that $\mathbf{M}_{g, \mu}^{\mathrm{rel}}\left(W_{t}, B_{r}\right)=\mathbf{M}_{g, \mu}^{\mathrm{rel}}\left(D_{r}\right)$ as sets. However, since the above construction works for analytic families of relative stable maps in $\mathbf{M}_{g, \mu}^{\mathrm{rel}}\left(W_{r}, B_{r}\right)$, this argument also shows that the two moduli spaces $\mathbf{M}_{g, \mu}^{\mathrm{rel}}\left(W_{r}, B_{r}\right)$ and $\mathbf{M}_{g, \mu}^{\mathrm{rel}}\left(D_{r}\right)$ are isomorphic as analytic schemes (stacks).

We now show that $\mathbf{M}_{g, \mu}^{\text {rel }}\left(D_{r}\right)$ is naturally an open subset of $\mathbf{M}_{g, \mu}^{\text {rel }}\left(\mathbf{P}^{1}\right)_{0}$. Let $(f, \Sigma)$ be a relative holomorphic map in $\mathbf{M}_{g, \mu}^{\mathrm{rel}}\left(D_{r}\right)$. We let $D_{r}^{\epsilon}$ be $\left\{(r-\epsilon)^{d}<|w| \leq r^{d}\right\}$, which is a neighborhood of $\partial D_{r} \subset D_{r}$. Since $f(\Sigma) \subset D_{r}, f(\partial \Sigma) \subset \partial D_{r}$ and $d_{k}>0$, the derivative of $f$ along $\partial \Sigma$ is nowhere vanishing. Note that $f$ is analytic up to the boundary by the reflection principle. Hence there is a positive $\epsilon>0$ so that $f^{-1}\left(D_{r}^{\epsilon}\right) \rightarrow D_{r}^{\epsilon}$ is a covering space. Furthermore, if we let $U_{k}$ be the connected component of $f^{-1}\left(D_{r}^{\epsilon}\right)$ containing $\partial_{k} \Sigma$, then $U_{k} \rightarrow D_{r}^{\epsilon}$ is a $d_{k}$-fold covering. Hence, there is a biholomorphic map

$$
\rho_{k}:\left\{(r-\epsilon)^{-1}>\left|z_{k}\right|>r^{-1}\right\} \longrightarrow U_{k}
$$

so that $f \circ \rho_{k}$ is given by $w=z_{k}^{-d_{k}}$. We then attach the disk $\left\{\left|z_{k}\right|<(r-\epsilon)^{-1}\right\}$ to $\Sigma$ using the isomorphism $\rho_{k}$. We let $C$ be the complete nodal curve resulting from 
attaching $n$ disks to the $n$ holes, following the procedure described. Then the map $f: \Sigma \rightarrow D_{r}$ extends to a holomorphic map $C \rightarrow \mathbf{P}^{1}$, which we denote by $\bar{f}$, so that $\bar{f}$ restricting to the disk $\left\{\left|z_{k}\right|<(r-\epsilon)^{-1}\right\}$ is given by $w=z_{k}^{-d_{k}}$. Thus $(\bar{f}, C)$ is a relative stable morphism in $\mathbf{M}_{g, \mu}^{\mathrm{rel}}\left(\mathbf{P}^{1}\right)$. It is straightforward to check that any two such extensions (to $(f, C)$ ) are equivalent. This way we obtain a map

$$
\mathbf{M}_{g, \mu}^{\mathrm{rel}}\left(D_{r}\right) \longrightarrow \mathbf{M}_{g, \mu}^{\mathrm{rel}}\left(\mathbf{P}^{1}\right) .
$$

This map is obviously injective and the image is an open subset in $\mathbf{M}_{g, \mu}^{\mathrm{rel}}\left(\mathbf{P}^{1}\right)$. On the other hand, this construction works for any analytic family of relative stable maps in $\mathbf{M}_{g, \mu}^{\text {rel }}\left(D_{r}\right)$. This shows that 2-5 is an open embedding. In the following, we will view $\mathbf{M}_{g, \mu}^{\text {rel }}\left(D_{r}\right)$ as an open subset of $\mathbf{M}_{g, \mu}^{\text {rel }}\left(\mathbf{P}^{1}\right)$. It is straightforward to check that, for $r>r^{\prime}$, $\mathbf{M}_{g, \mu}^{\mathrm{rel}}\left(D_{r}\right) \subset \mathbf{M}_{g, \mu}^{\mathrm{rel}}\left(\mathbf{P}^{1}\right)$ is contained in $\mathbf{M}_{g, \mu}^{\mathrm{rel}}\left(D_{r^{\prime}}\right) \subset \mathbf{M}_{g, \mu}^{\mathrm{rel}}\left(\mathbf{P}^{1}\right)$. When we take the union of all these open subsets, we obtain

$$
\bigcup_{r>0} \mathbf{M}_{g, \mu}^{\mathrm{rel}}\left(D_{r}\right)=\mathbf{M}_{g, \mu}^{\mathrm{rel}}\left(\mathbf{P}^{1}\right)_{0} .
$$

\section{Obstruction theory of $\mathbf{M}_{g, \mu}^{\mathrm{rel}}\left(W_{r}, B_{r}\right)$}

We now investigate the obstruction theory of $\mathbf{M}_{g, \mu}^{\mathrm{rel}}\left(W_{r}\right)$. First, since $\mathbf{M}_{g, \mu}^{\mathrm{rel}}\left(W_{r}\right)$ is identical to $\mathbf{M}_{g, \mu}^{\mathrm{rel}}\left(B_{r}\right)$ as analytic schemes (stacks), the space of the first order deformations of $f \in \mathbf{M}_{g, \mu}^{\mathrm{rel}}\left(W_{r}, B_{r}\right)$ is naturally isomorphic to the space of the first order deformations of $f$ as an elements in $\mathbf{M}_{g, \mu}^{\mathrm{rel}}\left(D_{r}\right)$. As to the obstruction, since there are no first order deformations of $f \in \mathbf{M}_{g, \mu}^{\mathrm{rel}}\left(W_{r}\right)$ along the fiber direction of $W_{r} \rightarrow D_{r}$, the obstruction classes to extending families in $\mathbf{M}_{g, \mu}^{\mathrm{rel}}\left(W_{r}\right)$ are exactly the obstruction classes to extending them as families in $\mathbf{M}_{g, \mu}^{\mathrm{rel}}\left(D_{r}\right)$. Furthermore, the obstruction spaces (sheaves) to extending families in $\mathbf{M}_{g, \mu}^{\text {rel }}\left(W_{r}\right)$ will then be the direct sum of the obstruction spaces (sheaves) to extending them in $\mathbf{M}_{g, \mu}^{\mathrm{rel}}\left(D_{r}\right)$ with the obstruction spaces (sheaves) to deforming them along the fiber directions of $W_{r}$. Let $V$ be the obstruction bundle to extending families along the fiber directions of $W_{r}$. According to the theory of virtual moduli cycles (Li-Tian $[14,15])$, the virtual moduli cycle of $\mathbf{M}_{g, \mu}^{\mathrm{rel}}\left(W_{r}\right)$ is the top Chern class of the obstruction bundle $V$ paired with the virtual moduli cycle of $\mathbf{M}_{g, \mu}^{\mathrm{rel}}\left(D_{r}\right)$.

We now derive this obstruction bundle $V$. Let $\left(f_{r}, \Sigma_{r}\right) \in \mathbf{M}_{g, \mu}^{\mathrm{rel}}\left(W_{r}\right)$ be any relative map. We let $(f, C)$ be the canonical extension of $\left(f_{r}, \Sigma_{r}\right)$ to $C \rightarrow \mathbf{P}^{1}$ constructed before. For any $r^{\prime} \geq r$ we let $\Sigma_{r^{\prime}}=C \cap f^{-1}\left(D_{r^{\prime}}\right)$. Then $f_{r^{\prime}}=\left.f\right|_{\Sigma_{r^{\prime}}}: \Sigma_{r^{\prime}} \rightarrow D_{r^{\prime}}$ is an element in $\mathbf{M}_{g, \mu}^{\mathrm{rel}}\left(D_{r^{\prime}}\right)=\mathbf{M}_{g, \mu}^{\mathrm{rel}}\left(W_{r^{\prime}}\right)$. In the following we will identify the obstruction space $V\left(f_{r}\right)$ 
to deforming $f_{r}$ along the fiber directions of $W_{r}$. As expected, the space $V\left(f_{r}\right)$ will be canonically isomorphic to $V\left(f_{r^{\prime}}\right)$.

We now derive the space $V\left(f_{r}\right)$. We first consider the case where $f=1$ and $C$ is smooth. We pick a sufficiently small $\epsilon>0$ so that $U_{r, 1}=f^{-1}\left(D_{r}^{\epsilon}\right) \subset \Sigma_{r}$ is isomorphic to $\left\{r^{-1} \leq|z|<r^{-1}+\epsilon\right\}$ and that $\left.f\right|_{U_{r, 1}}: U_{r, 1} \rightarrow \mathbf{C}$ is defined by $w=z^{-d}$. Now we let $U_{r, 0}=\Sigma_{r}-\partial \Sigma_{r}$. Then $U_{r, 0}$ and $U_{r, 1}$ are an open covering of $\Sigma_{r}$ with

$$
U_{r, 1} \cap U_{r, 0} \cong\left\{r^{-1}<|z|<r^{-1}+\epsilon\right\} .
$$

Our first task is to describe the deformations of $\left.f_{r}\right|_{U_{r, 0}}$ and $\left.f_{r}\right|_{U_{r, 1}}$ along the fiber directions of $W_{r}$. Clearly, deformations of $\left.f_{r}\right|_{U_{r, 0}}$ are given by the space of sections

$$
s_{0} \in \Gamma\left(U_{r, 0}, f_{r}^{*} L_{1} \oplus f_{r}^{*} L_{2}\right),
$$

whereas deformations of $\left.f_{r}\right|_{U_{r, 1}}$ are given by the sections

$$
s_{1} \in \Gamma\left(U_{r, 1}, f_{r}^{*} L_{1} \oplus f_{r}^{*} L_{2}\right)
$$

subject to the boundary condition $2-1$. We now investigate the boundary condition in detail. Using the distinguished basis $e_{1}$ and $e_{2}$, we can express $s_{1}$ as

$$
s_{1}=u_{1}(z) f_{r}^{*} e_{1}+u_{2}(z) f_{r}^{*} e_{2},
$$

where $u_{1}$ and $u_{2}$ are continuous functions over $U_{r, 1}$ that are holomorphic in the interior of $U_{r, 1}$. We let $u_{1}(z)=\sum_{k=-\infty}^{\infty} a_{k} z^{k}$ and $u_{2}(z)=\sum_{k=-\infty}^{\infty} b_{k} z^{k}$ be the Laurent series expansions of $u_{1}$ and $u_{2}$. Then the boundary condition 2-1 (after substituting $z$ by $\left.r^{-1} e^{i \theta}\right)$ is equivalent to

$$
\sum_{k=-\infty}^{\infty} a_{k} r^{-k} e^{i k \theta}=\left(r^{-1} e^{-i \theta}\right)^{-d} \sum_{k=-\infty}^{\infty} \bar{b}_{k} r^{-k} e^{-i k \theta}, \theta \in \mathbb{R} .
$$

This forces

$$
a_{k} r^{-k}=\bar{b}_{d-k} r^{k}
$$

for all $k \in \mathbb{Z}$. When $r \rightarrow \infty$, the above relations reduce to

$$
a_{k}=0 \text { for } k<0 ; \quad b_{k}=0 \text { for } k<d \quad \text { and } \quad a_{0}=\bar{b}_{d} .
$$

We let

$$
\left.\Gamma\left(U_{r, 1}, f_{r}^{*} L_{1} \oplus f_{r}^{*} L_{2}\right)\right)_{r}=\left\{s \in \Gamma\left(U_{r, 0}, f_{r}^{*} L_{1} \oplus f_{r}^{*} L_{2}\right) \mid s \text { satisfies 3-1 }\right\},
$$

and

$$
\Gamma\left(U_{r, 1}, f_{r}^{*} L_{1} \oplus f_{r}^{*} L_{2}\right)_{\infty}=\left\{s \in \Gamma\left(U_{r, 1}, f_{r}^{*} L_{1} \oplus f_{r}^{*} L_{2}\right) \mid s \text { satisfies 3-2 }\right\}
$$


According to the receipt of the obstruction theory, the obstruction space to deforming

$$
f_{r}:\left(\Sigma_{r}, \partial \Sigma_{r}\right) \longrightarrow\left(W_{r}\right)
$$

along the fiber directions of $W_{r}$ is the cokernel of

$$
\begin{aligned}
\Gamma\left(U_{r, 1}, f_{r}^{*} L_{1} \oplus f_{r}^{*} L_{2}\right)_{r} \oplus \Gamma\left(U_{r, 0}, f_{r}^{*} L_{1} \oplus f_{r}^{*} L_{2}\right) \longrightarrow \\
\Gamma\left(U_{r, 0} \cap U_{r, 1}, f_{r}^{*} L_{1} \oplus f_{r}^{*} L_{2}\right) .
\end{aligned}
$$

Using the relation 3-1 it is easy to see that the above cokernel is canonically isomorphic to the cokernel of

$$
\begin{aligned}
\Gamma\left(U_{r, 1}, f_{r}^{*} L_{1} \oplus f_{r}^{*} L_{2}\right)_{\infty} \oplus \Gamma\left(U_{r, 0}, f_{r}^{*} L_{1} \oplus f_{r}^{*} L_{2}\right) \longrightarrow \\
\Gamma\left(U_{r, 0} \cap U_{r, 1}, f_{r}^{*} L_{1} \oplus f_{r}^{*} L_{2}\right) .
\end{aligned}
$$

Our next step is to repress this cokernel in terms of some cohomology groups of line bundles over $C$. For this we need to extend the line bundles $f_{r}^{*} L_{1}$ and $f_{r}^{*} L_{2}$ to line bundles over $C$. We extend both line bundle to trivial line bundles over $C$ so that the constant sections $e_{i}$ of $L_{i}$ pull back to a constant section of $\mathcal{O}_{C}$. With this choice of extensions, $\Gamma\left(U_{r, 1}, f_{r}^{*} L_{1} \oplus f_{r}^{*} L_{2}\right)_{\infty}$ then is canonically isomorphic to

$$
\Gamma\left(\mathcal{O}_{\mathcal{U}_{1}}(-x)\right) \oplus \Gamma\left(\mathcal{O}_{\mathcal{U}_{1}}(-d x)\right) \text {. }
$$

Here $\mathcal{U}_{1}$ is the disk $\{|z|<\epsilon\} \subset C, x \in \bar{U}_{r, 1}$ is the point ramified over $q_{\infty} \in \mathbf{P}^{1}$ and $\mathcal{U}_{0}=C-x$. Hence the cokernel of 3-5 becomes the cokernel of

$$
\begin{aligned}
& \Gamma\left(\mathcal{U}_{1}, \mathcal{O}_{C}(-x) \oplus \mathcal{O}_{C}(-d x)\right) \oplus \Gamma\left(\mathcal{U}_{0}, \mathcal{O}_{C}(-x) \oplus \mathcal{O}_{C}(-d x)\right) \longrightarrow \\
& \Gamma\left(\mathcal{U}_{0} \cap \mathcal{U}_{1}, \mathcal{O}_{C}(-x) \oplus \mathcal{O}_{C}(-d x)\right),
\end{aligned}
$$

which by the definition of Čech cohomology is

$$
H^{1}\left(\mathcal{O}_{C}(-x)\right) \oplus H^{1}\left(\mathcal{O}_{C}(-d x)\right) .
$$

This shows that the obstruction space $V\left(f_{r}\right)$ of $f_{r} \in \mathbf{M}_{g, \mu}^{\mathrm{rel}}\left(W_{r}\right)$ is the cohomology group $3-9$.

The vector spaces $V\left(f_{r}\right)$ over $f_{r} \in \mathbf{M}_{g, \mu}^{\mathrm{rel}}\left(W_{r}\right)$ form a vector bundles over $\mathbf{M}_{g, \mu}^{\mathrm{rel}}\left(W_{r}\right)$. Since $V\left(f_{r}\right)$ is independent of $r$, this vector bundle extends to $\mathbf{M}_{g, \mu}^{\mathrm{rel}}\left(\mathbf{P}^{1}\right)_{0}$ via the same formula. We denote this vector bundle over $\mathbf{M}_{g, \mu}^{\mathrm{rel}}\left(\mathbf{P}^{1}\right)_{0}$ by $V$.

Proposition 3.1 Let $f_{r} \in \mathbf{M}_{g, \mu}^{\text {rel }}\left(\mathbf{P}^{1}\right)$ be any relative stable morphism with $C$ the domain of $f$ and let $D_{\mathbf{d}}=d_{1} x_{1}+\cdots+d_{h} x_{h}$ be the distinguished divisor in $C$. We let $D=x_{1}+\cdots+x_{h}$ and let $V$ be the vector bundle over $\mathbf{M}_{g, \mu}^{\text {rel }}\left(\mathbf{P}^{1}\right)$ whose fibers over $f$ are

$$
V(f)=H^{1}\left(\mathcal{O}_{C}(-D) \oplus \mathcal{O}_{C}\left(-D_{\mathbf{d}}\right)\right)
$$


Then the obstruction bundle to deforming maps in $\mathbf{M}_{g, \mu}^{\text {rel }}\left(W_{r}\right)$ along the fiber directions of $W_{r}$ is given by the vector bundle $\left.V\right|_{\mathbf{M}_{g, \mu}^{\text {rel }}\left(W_{r}\right)}$.

Proof The proof is exactly the same as the case $h=1$ and $\Sigma_{r}$ is smooth, except that, in deriving this cohomology space, we need to cover $\Sigma_{r}$ by more than two open subsets.

According to the general principle of virtual moduli cycles, the expected (virtual) number of maps in $\mathbf{M}_{g, \mu}^{\mathrm{rel}}\left(W_{r}\right)$ should be

$$
\int_{\left[\mathbf{M}_{g, \mu}^{\mathrm{rel}}\left(W_{r}\right)\right]^{\mathrm{vir}}} c_{\mathrm{top}}(V)
$$

Here $\left[\mathbf{M}_{g, \mu}^{\mathrm{rel}}\left(W_{r}\right)\right]^{\mathrm{vir}}$ is the virtual moduli cycle of $\mathbf{M}_{g, \mu}^{\mathrm{rel}}\left(W_{r}\right)$ and $c_{\text {top }}$ is the top Chern class of a vector bundle. Clearly, this integration is not well defined mathematically because $\mathbf{M}_{g, \mu}^{\mathrm{rel}}\left(W_{r}\right)$ is not compact. To make sense of this integral we need to compactify $\mathbf{M}_{g, \mu}^{\mathrm{rel}}\left(W_{r}\right)$ and then extend $V$. Since $\mathbf{M}_{g, \mu}^{\mathrm{rel}}\left(W_{r}\right)$ is naturally an open subset of $\mathbf{M}_{g, \mu}^{\mathrm{rel}}\left(\mathbf{P}^{1}\right)$, we can use $\mathbf{M}_{g, \mu}^{\text {rel }}\left(\mathbf{P}^{1}\right)$ to compactify $\mathbf{M}_{g, \mu}^{\text {rel }}\left(\mathbf{P}^{1}\right)_{0}$. It was proved by the first author of the present paper that $\mathbf{M}_{g, \mu}^{\mathrm{rel}}\left(\mathbf{P}^{1}\right)$ is a proper Deligne-Mumford stack, and that it admits a perfect obstruction theory and thus comes with a natural choice of virtual moduli cycles, denoted by $\left[\mathbf{M}_{g, \mu}^{\mathrm{rel}}\left(\mathbf{P}^{1}\right)\right]^{\mathrm{vir}}$.

Extending $V$ to $\mathbf{M}_{g, \mu}^{\text {rel }}\left(\mathbf{P}^{1}\right)$ needs more work. There is an obvious extension as follows: Let $f \in \mathbf{M}_{g, \mu}^{\text {rel }}\left(\mathbf{P}^{1}\right)$ be any relative stable morphism with domain $C$ and distinguished divisor $D_{\mathbf{d}}$ as before. The the vector bundle $\tilde{V}$ whose fiber over $f$ is the vector space $V(f)$, is a vector bundle over $\mathbf{M}_{g, \mu}^{\mathrm{rel}}\left(\mathbf{P}^{1}\right)$. However, there probably are other extensions, and at the moment we have no reason as to which one is a natural choice of extension. Note that different extensions may give different numerical answers to the integral. The choice of the right extension requires a detailed analysis of the obstruction theory of $f$ near the boundary of $\mathbf{M}_{g, \mu}^{\mathrm{rel}}\left(W_{r}\right)$, and we will address this issue in our future research.

\section{Relative stable morphisms and localization}

In this and the next sections, we will use localization to evaluate the integral 3-10. In Section 2, we defined an $S^{1}$-action on $W_{r}$ via the rule

$$
(w, u, v)^{t}=\left(t^{-1} w, t u, v\right), \quad t=e^{2 \pi i \theta} \quad \text { where } \theta \in \mathbb{R} .
$$


This action extends to the total space $\mathbf{1}_{\mathbf{C}} \oplus \mathbf{1}_{\mathbf{C}}$ via the same rule. As we mentioned before, this action preserves the boundary condition $B_{r} \subset W_{r}$, and thus it is reasonable to expect that localization using this $S^{1}$-action will give us the correct answer.

Let $\mathbf{M}_{g, \mu}^{\text {rel }}\left(\mathbf{P}^{1}\right)_{0}$ be the moduli space of ordinary relative stable morphisms defined before. The given $S^{1}$-action on $W_{r}$ induces a natural $S^{1}$-action on $\mathbf{M}_{g, \mu}^{\text {rel }}\left(\mathbf{P}^{1}\right)_{0}$. In this section, we will study the fixed loci of the induced $S^{1}$-action on $\mathbf{M}_{g, \mu}^{\mathrm{rel}}\left(\mathbf{P}^{1}\right)_{0}$ and its related information required in the later computation. Let $f \in \mathbf{M}_{g, \mu}^{\text {rel }}\left(\mathbf{P}^{1}\right)_{0}$ be any relative stable map. As before, we denote by $\left(C, D_{\mathbf{d}}\right)$ the pair of the domain of $f$ and the distinguished divisor of $f$. Since $f \in \mathbf{M}_{g, \mu}^{\text {rel }}\left(\mathbf{P}^{1}\right)_{0}$, the codomain of $f$ is $\mathbf{P}^{1}$ and $f^{-1}\left(q_{\infty}\right)=D_{\mathbf{d}}$. We recall that if we denote by $g_{t}$ the $S^{1}$-action then

$$
g_{t}[w, 1]=[t w, 1] \quad \text { and } \quad g_{t *}(w)=g_{t^{-1}}^{*} w=t^{-1} w .
$$

If we use $\tilde{t}$ to denote the weight of the $S^{1}$-action, then the function $w$ has weight $-\tilde{t}$. The two fixed points of the $S^{1}$-action on $\mathbf{P}^{1}$ are $q_{0}=[0,1] \in \mathbf{P}^{1}$ and $q_{\infty}=[1,0] \in \mathbf{P}^{1}$.

In the above notation, considering source Riemann surfaces with one hole corresponds to setting $h=1$, and in the remainder of this section that is what we will do. For genus $g=0$, there is only one fixed point in $\mathbf{M}_{g, \mu}^{\mathrm{rel}}\left(\mathbf{P}^{1}\right)_{0}^{S^{1}}$ given by the map

$$
f: \mathbf{P}^{1} \rightarrow \mathbf{P}^{1}, \quad f([z, 1])=\left[z^{d}, 1\right] .
$$

For $g>0$, the fixed loci are the image of the embedding

$$
\mathbf{M}_{g, 1} \longrightarrow \mathbf{M}_{g, \mu}^{\mathrm{rel}}\left(\mathbf{P}^{1}\right)_{0}^{S^{1}}
$$

that sends any $\left(C_{2}, p\right) \in \mathbf{M}_{g, 1}$ to the relative stable morphism

$$
f:(C, d x) \longrightarrow \mathbf{P}^{1}
$$

defined as follows: First, the curve $C$ is the gluing of $\mathbf{P}^{1}$ with $C_{2}$ along the points $0 \in \mathbf{P}^{1}$ and $p \in C_{2}$; the restriction of $f$ to $C_{2}$ is the constant map sending $C_{2}$ to $q_{0}$ and the restriction of $f$ to $C_{1} \equiv \mathbf{P}^{1}$ is define by $[z, 1] \mapsto\left[z^{d}, 1\right]$. Since $w=z^{d}$ and the weight of $w$ is $-\tilde{t}$, the weight of the function $z$ is $-\tilde{t} / d$. For simplicity, in the following we will simply denote $\left.f\right|_{C_{i}}$ by $f_{i}$. We will use $p$ to denote the node in $C$.

If we consider the full moduli space $\mathbf{M}_{g, \mu}^{\text {rel }}\left(\mathbf{P}^{1}\right)$, however, there are other fixed loci of the $S^{1}$-action. As described in the previous section, a relative stable morphism $f \in \mathbf{M}_{g, \mu}^{\mathrm{rel}}\left(\mathbf{P}^{1}\right)$ is a morphism $f: C \rightarrow \mathbf{P}^{1}[m]$. The $S^{1}$-action extends to $\mathbf{P}^{1}[m]$ an $S^{1}-$ action on $\mathbf{M}_{g, \mu}^{\mathrm{rel}}\left(\mathbf{P}^{1}\right)$. We will denote the fixed loci of $\mathbf{M}_{g, \mu}^{\mathrm{rel}}\left(\mathbf{P}^{1}\right)$ contained in $\mathbf{M}_{g, \mu}^{\mathrm{rel}}\left(\mathbf{P}^{1}\right)_{0}$ by $\Theta_{I}$. The rest of the $S^{1}$-fixed loci of $\mathbf{M}_{g, \mu}^{\mathrm{rel}}\left(\mathbf{P}^{1}\right)$ will be denoted by $\Theta_{I I}$. 
Now let $N_{\Theta_{I}}$ be the equivariant normal bundle to the fixed loci $\Theta_{I}$. In this part, we will compute the equivariant Euler class $e\left(N_{\Theta_{I}}\right)$ of $\Theta_{I}$. Let $\left(f, C, D_{\mathbf{d}}\right) \in \Theta_{I}$ be any point. The tangent space and the obstruction space to deforming $f$ are given by the extension groups

$$
T^{\bullet}:=\operatorname{Ext}_{C}^{\bullet}\left(\left[f^{*} \Omega_{\mathbf{P}^{1}}\left(\log q_{\infty}\right) \rightarrow \Omega_{C}(x)\right], \mathcal{O}_{C}\right),
$$

which fit into the long exact sequence

$$
\begin{aligned}
0 & \longrightarrow \operatorname{Ext}_{C}^{0}\left(\Omega_{C}(x), \mathcal{O}_{C}\right) \longrightarrow \operatorname{Ext}_{C}^{0}\left(f^{*} \Omega_{\mathbf{p}^{1}}\left(\log q_{\infty}\right), \mathcal{O}_{C}\right) \longrightarrow T^{1} \longrightarrow \\
& \longrightarrow \operatorname{Ext}_{C}^{1}\left(\Omega_{C}(x), \mathcal{O}_{C}\right) \longrightarrow \operatorname{Ext}_{C}^{1}\left(f^{*} \Omega_{\mathbf{P}^{1}}\left(\log q_{\infty}\right), \mathcal{O}_{C}\right) \longrightarrow T^{2} \longrightarrow 0 .
\end{aligned}
$$

From this information, we can obtain the equivariant Euler class $e\left(N_{\Theta_{I}}\right)$. Following the notation of Graber and Pandharipande [5], it is given by

$$
e\left(N_{\Theta_{I}}\right)=\frac{e\left(B_{I I}^{m}\right) e\left(B_{I V}^{m}\right)}{e\left(B_{I}^{m}\right) e\left(B_{V}^{m}\right)}
$$

where $B_{i}^{m}$ denotes the moving part of the $i^{\text {th }}$ term in the sequence $4-1$.

We now consider the case $g>0$. In this case $C=C_{1} \cup C_{2}$ with the node $p$. We let $i_{1}: C_{1} \rightarrow C$ and $i_{2}: C_{2} \rightarrow C$ be inclusion maps. Then

$$
\Omega_{C}=i_{1 *} \Omega_{C_{1}} \oplus i_{2 *} \Omega_{C_{2}} \oplus \mathbb{C}_{p} .
$$

Then

$$
\begin{aligned}
\operatorname{Ext}_{C}^{0}\left(\Omega_{C}(x), \mathcal{O}_{C}\right) & =\operatorname{Hom}_{C}\left(i_{1 *} \Omega_{C_{1}}(x), \mathcal{O}_{C}\right) \oplus \operatorname{Hom}_{C}\left(i_{2 *} \Omega_{C_{2}}(x), \mathcal{O}_{C}\right) \\
& \oplus \operatorname{Hom}_{C}\left(\mathbb{C}_{p}, \mathcal{O}_{C}\right) \\
& =\operatorname{Hom}_{C_{1}}\left(\Omega_{C_{1}}(x), \mathcal{O}_{C_{1}}(-p)\right)=\mathrm{H}_{C_{1}}^{0}\left(T_{C_{1}}(-p-x)\right) .
\end{aligned}
$$

It is easy to see that the second arrow in $4-1$ is injective. Thus we can take $e\left(B_{I}^{m}\right)=1$ while keeping track of the term in $e\left(B_{I I}^{m}\right)$ to be cancelled.

As to the term $e\left(B_{I I}^{m}\right)$, we notice that

$$
\operatorname{Ext}_{C}^{0}\left(f^{*} \Omega_{\mathbf{P}^{1}}\left(\log q_{\infty}\right), \mathcal{O}_{C}\right)=\mathrm{H}_{C_{1}}^{0}\left(f_{1}^{*} T_{\mathbf{P}^{1}}(-d \cdot x)\right) .
$$

$\mathrm{H}_{C_{1}}^{0}\left(f_{1}^{*} T_{\mathbf{P}^{1}}(-d x)\right)$ has dimension $d+1$ and its basis is given by

$$
\left\{\frac{\partial}{\partial w}, z \frac{\partial}{\partial w}, z^{2} \frac{\partial}{\partial w}, \ldots, z^{d-1} \frac{\partial}{\partial w}, z^{d} \frac{\partial}{\partial w}\right\}
$$

with weights

$$
\left\{\tilde{t}, \frac{d-1}{d} \tilde{t}, \frac{d-2}{d} \tilde{t}, \ldots, \frac{1}{d} \tilde{t}, 0\right\}
$$


Thus, modulo the weight-zero piece which was cancelled by the second arrow in 4-1, we have

$$
\frac{e\left(B_{I I}^{m}\right)}{e\left(B_{I}^{m}\right)}=\prod_{j=0}^{d-1} \frac{d-j}{d} \tilde{t}=\frac{d !}{d^{d}} \tilde{t}^{d} .
$$

We now look at the term $e\left(B_{I V}^{m}\right)$. We have

$$
\begin{aligned}
\operatorname{Ext}_{C}^{1}\left(\Omega_{C}(x), \mathcal{O}_{C}\right) & =\operatorname{Ext}_{C}^{0}\left(\mathcal{O}_{C}, \Omega_{C}(x) \otimes \omega_{C}\right)^{\vee} \\
& =\operatorname{Ext}_{C_{2}}^{0}\left(\mathcal{O}_{C_{2}}, \omega_{C_{2}}^{\otimes 2}(p)\right)^{\vee} \oplus \operatorname{Ext}_{C}^{0}\left(\mathcal{O}_{C}, \mathbb{C}_{p} \otimes \omega_{C}\right)^{\vee} \\
& =\operatorname{Ext}_{C_{2}}^{1}\left(\Omega_{C_{2}}(p), \mathcal{O}_{C_{2}}\right) \oplus T_{C_{1}, p}^{\vee} \otimes T_{C_{2}, p}^{\vee} .
\end{aligned}
$$

Since $f\left(C_{2}\right)=q_{0}, \operatorname{Ext}_{C_{2}}^{1}\left(\Omega_{C_{2}}(p), \mathcal{O}_{C_{2}}\right)$ lies in the fixed part of $\operatorname{Ext}_{C}^{1}\left(\Omega_{C}(x), \mathcal{O}_{C}\right)^{S^{1}}$. On the other hand, $T_{C_{1}, p}^{\vee}$ has weight $\tilde{t} / d$ while $T_{C_{2}, p}^{\vee}$ has weight 0 . Hence

$$
e\left(B_{I V}^{m}\right)=\frac{1}{d} \tilde{t}-\psi=\frac{\tilde{t}-d \cdot \psi}{d},
$$

where $\psi=c_{1}\left(\mathcal{L}_{p}\right)$ and $\mathcal{L}_{p} \rightarrow \bar{M}_{g, 1}$ is the cotangent line bundle whose fiber over $\left(C_{2}, p\right)$ is $T_{C_{2}, p}^{\vee}$.

Finally, it is direct to compute that

$$
\operatorname{Ext}_{C}^{1}\left(f^{*} \Omega_{\mathbf{p}^{1}}\left(\log q_{\infty}\right), \mathcal{O}_{C}\right)=\mathrm{H}_{C_{2}}^{1}\left(\mathcal{O}_{C_{2}}\right) \otimes T_{q_{0}} \mathbf{P}^{1} .
$$

Note that $\mathrm{H}_{C_{2}}^{1}\left(\mathcal{O}_{C_{2}}\right)$ gives the dual of the Hodge bundle $E$ on $\bar{M}_{g, 1}$. Hence $e\left(B_{V}^{m}\right)$ is the top Chern class of the bundle $E^{\vee} \otimes T_{q_{0}} \mathbf{P}^{1}$. Since the representation of the $S^{1}$-action induced on $T_{q_{0}} \mathbf{P}^{1}$ has weight $\tilde{t}$, we obtain

$$
\begin{aligned}
e\left(B_{V}^{m}\right) & =c_{\mathrm{top}}\left(E^{\vee} \otimes T_{q_{0}} \mathbf{P}^{1}\right) \\
& =\left(\tilde{t}^{g}+c_{1}\left(E^{\vee}\right) \tilde{t}^{g-1}+c_{2}\left(E^{\vee}\right) \tilde{t}^{g-2}+\cdots+c_{g}\left(E^{\vee}\right)\right) .
\end{aligned}
$$

Hence, we arrive at the following result:

$$
\begin{aligned}
\frac{1}{e\left(N_{\Theta_{I}}\right)}=\frac{d^{d}}{d !} \tilde{t}^{-d}\left(\frac{d}{\tilde{t}-d \cdot \psi}\right) & \\
& \left(\tilde{t}^{g}+c_{1}\left(E^{\vee}\right) \tilde{t}^{g-1}+c_{2}\left(E^{\vee}\right) \tilde{t}^{g-2}+\cdots+c_{g}\left(E^{\vee}\right)\right) .
\end{aligned}
$$

In the case $g=0$, a similar computation yields

$$
\frac{1}{e\left(N_{\Theta_{I}}\right)}=\frac{1}{d} \cdot \frac{d^{d}}{d !} \tilde{t}^{1-d} .
$$




\section{Localization of the integral}

We now evaluate the contribution of the integral

$$
\int_{\left[\mathbf{M}_{g, \mu}^{\mathrm{rel}}\left(\mathbf{P}^{1}\right)\right]^{\mathrm{vir}}} c_{\mathrm{top}}(V)
$$

at the fixed loci $\Theta_{I}$ using the $S^{1}$-action given before. Here $V$ is the obstruction vector bundle defined in Proposition 3.1. Using the localization theorem [5], we have

$$
\left[\int_{\left[\mathbf{M}_{g, \mu}^{\mathrm{rel}}\left(\mathbf{P}^{1}\right)\right]^{\mathrm{vir}}} c_{\mathrm{top}}(V)\right]_{\Theta_{I}}=\left[\frac{1}{\left|\mathrm{~A}_{\Theta_{I}}\right|} \int_{\Theta_{I}} \frac{\iota^{*}\left(c_{\mathrm{top}}(V)\right)}{e\left(N_{\Theta_{I}}\right)}\right]_{0},
$$

where $\iota$ is the inclusion $\Theta_{I} \rightarrow \mathbf{M}_{g, \mu}^{\text {rel }}\left(\mathbf{P}^{1}\right)$ and $A_{\Theta_{I}}$ is a automorphism group defined as in [5].

We notice that by Riemann-Roch theorem,

$$
\begin{aligned}
\operatorname{dim}_{\mathbb{C}} \mathbf{M}_{g, \mu}^{\mathrm{rel}}\left(\mathbf{P}^{1}\right) & =2 d+(1-g)\left(\operatorname{dim}_{\mathbb{C}} \mathbf{P}^{1}-3\right)-(\operatorname{deg}(\mathbf{d})-\ell(\mathbf{d})) \\
& =2 g-2+h+d \\
& =\operatorname{dim}_{\mathbb{C}} \mathrm{H}^{1}\left(C, \mathcal{O}_{C}\left(-D_{\mathbf{d}}\right) \oplus \mathcal{O}_{C}(-D)\right) .
\end{aligned}
$$

In this section, we focus on source Riemann surfaces with one hole $(h=1)$, in which case we need to find the equivariant Chern class $c_{\text {top }}(V)$ where $V$ is defined as follows. Let $f \in \Theta_{I}$ be a point as before. Then the fiber of $V$ over $f$ is

$$
H^{1}\left(\mathcal{O}_{C}(-x)\right) \oplus H^{1}\left(\mathcal{O}_{C}(-d x)\right) .
$$

For simplicity, we denote by $V_{1}$ the subbundle of $V$ whose fibers over $f$ are the vector space $H^{1}\left(\mathcal{O}_{C}(-x)\right)$, and by $V_{2}$ the subbundle of $V$ whose fibers over $f$ are $H^{1}\left(\mathcal{O}_{C}(-d x)\right)$. The line bundle $\mathcal{O}_{C}$ in the first cohomology group has weight $-\tilde{t}$ while the line bundle $\mathcal{O}_{C}$ in the second cohomology group has weight 0 . We will work out the genus-zero and higher genus cases separately.

We first look at the genus $g=0$ case. In this case, $\mathrm{H}^{1}\left(C, \mathcal{O}_{C}(-x)\right)=0$ and hence $V=V_{2}$. To analyze the contribution from $\mathrm{H}^{1}\left(C, \mathcal{O}_{C}(-d x)\right)$, we use the exact sequence

$$
0 \longrightarrow \mathcal{O}_{C}(-d x) \longrightarrow \mathcal{O}_{C} \longrightarrow \mathcal{O}_{d x} \longrightarrow 0
$$

and the induced cohomology exact sequence

$$
0 \longrightarrow \mathrm{H}^{0}\left(\mathcal{O}_{C}\right) \longrightarrow \mathrm{H}^{0}\left(\mathcal{O}_{d x}\right) \longrightarrow \mathrm{H}^{1}\left(\mathcal{O}_{C}(-d x)\right) \longrightarrow 0 .
$$


A basis of $\mathrm{H}^{0}\left(\mathcal{O}_{C}\right)$ is just $\{1\}$ and that of $\mathrm{H}^{0}\left(\mathcal{O}_{d x}\right)$ is $\left\{1, z^{-1}, z^{-2}, \ldots, z^{-(d-1)}\right\}$. Hence a basis of $\mathrm{H}^{1}\left(\mathcal{O}_{C}(-d x)\right)$ is

$$
\left\{\frac{1}{z}, \frac{1}{z^{2}}, \ldots, \frac{1}{z^{d-1}}\right\}
$$

with weights

$$
\left\{\frac{1}{d} \tilde{t}, \frac{2}{d} \tilde{t}, \ldots, \frac{d-1}{d} \tilde{t}\right\} .
$$

Thus the top Chern class of the obstruction bundle $V$ is

$$
c_{\text {top }}(V)=c_{\text {top }}\left(V_{2}\right)=\prod_{j=1}^{d-1} \frac{j}{d} \tilde{t}=\frac{(d-1) !}{d^{d-1}} \tilde{t}^{d-1} .
$$

In the cases with $g>0, C$ is a union of the two irreducible components $C_{1}=\mathbf{P}^{1}$ and $C_{2}=\Sigma_{g}$ intersecting at the node $p$. Then there is the normalization exact sequence

$$
0 \longrightarrow \mathcal{O}_{C}(-d x) \longrightarrow \mathcal{O}_{C_{1}}(-d x) \oplus \mathcal{O}_{C_{2}} \longrightarrow \mathcal{O}_{p} \longrightarrow 0,
$$

which gives the following exact sequence of cohomology:

$$
\begin{aligned}
& 0 \longrightarrow \mathrm{H}_{C_{2}}^{0}\left(\mathcal{O}_{C_{2}}\right) \stackrel{\cong}{\cong} \mathrm{H}_{C}^{0}\left(\mathcal{O}_{p}\right) \longrightarrow \mathrm{H}_{C}^{1}\left(\mathcal{O}_{C}(-d x)\right) \longrightarrow \\
& \mathrm{H}_{C_{1}}^{1}\left(\mathcal{O}_{C_{1}}(-d x)\right) \oplus \mathrm{H}_{C_{2}}^{1}\left(\mathcal{O}_{C_{2}}\right) \longrightarrow 0 .
\end{aligned}
$$

So $\mathrm{H}^{1}\left(C, \mathcal{O}_{C}(-d x)\right)$ is given by

$$
\mathrm{H}^{1}\left(C, \mathcal{O}_{C}(-d x)\right)=\mathrm{H}^{1}\left(C_{1}, \mathcal{O}_{C_{1}}(-d x)\right) \oplus \mathrm{H}^{1}\left(C_{2}, \mathcal{O}_{C_{2}}\right) .
$$

We have already computed the contribution of $\mathrm{H}^{1}\left(C_{1}, \mathcal{O}_{C_{1}}(-d x)\right)$ to the equivariant Chern class $c_{\text {top }}\left(V_{2}\right)$. Since $\mathcal{O}_{C_{2}}$ in 5-5 has weight zero and there is no twisting, the contribution from $\mathrm{H}^{1}\left(C_{2}, \mathcal{O}_{C_{2}}\right)$ to $c_{\text {top }}(V)$ is $c_{g}\left(E^{\vee}\right)=(-1)^{g} c_{g}(E)$. Hence the contribution from $\mathrm{H}^{1}\left(C, \mathcal{O}_{C}(-d x)\right)$ to the equivariant top Chern class $c_{\text {top }}\left(V_{2}\right)$ is

$$
(-1)^{g} \frac{(d-1) !}{d^{d-1}} c_{g}(E) \tilde{t}^{d-1} \text {. }
$$

In a similar spirit, we can use the same line of reasoning to get

$$
\mathrm{H}^{1}\left(C, \mathcal{O}_{C}(-x)\right)=\mathrm{H}^{1}\left(C_{1}, \mathcal{O}_{C_{1}}(-x)\right) \oplus \mathrm{H}^{1}\left(C_{2}, \mathcal{O}_{C_{2}}\right) .
$$

As discussed in the genus-zero case, $\mathrm{H}^{1}\left(C_{1}, \mathcal{O}_{C_{1}}(-x)\right)$ has dimension zero and does not contribute. To compute the contribution from $\mathrm{H}^{1}\left(C_{2}, \mathcal{O}_{C_{2}}\right)$, we recall that, as mentioned in the beginning of this section, $\mathcal{O}_{C_{2}}$ in the present case has weight $-\tilde{t}$. Hence

$$
c_{\text {top }}\left(V_{1}\right)=(-1)^{g}\left(\tilde{t}^{g}-c_{1}\left(E^{\vee}\right) \tilde{t}^{g-1}+c_{2}\left(E^{\vee}\right) \tilde{t}^{g-2}+\cdots+(-1)^{g} c_{g}\left(E^{\vee}\right)\right)
$$




$$
=(-1)^{g}\left(\tilde{t}^{g}+c_{1}(E) \tilde{t}^{g-1}+c_{2}(E) \tilde{t}^{g-2}+\cdots+c_{g}(E)\right) .
$$

In summary, we have

$$
c_{\text {top }}(V)=\frac{(d-1) !}{d^{d-1}} c_{g}(E)\left(\tilde{t}^{g}+c_{1}(E) \tilde{t}^{g-1}+c_{2}(E) \tilde{t}^{g-2}+\cdots+c_{g}(E)\right) \tilde{t}^{d-1} .
$$

We are now ready to evaluate the contribution from $\Theta_{I}$ to the integral 5-1 in the case $h=1$. We begin with the case $g=0$.

After taking into account the automorphism group $A_{\Theta_{I}}$, which has order $d$, the genuszero answer is given by multiplying the expressions in 4-9 and 5-3. This gives the virtual number in the genus-zero case invariant

$$
\frac{1}{d} \cdot \frac{1}{d} \frac{d^{d}}{d !} \tilde{t}^{1-d} \cdot \frac{(d-1) !}{d^{d-1}} \tilde{t}^{d-1}=\frac{1}{d^{2}},
$$

which agrees with $1-3$.

We now consider the cases $g>0$. We need to use the results in 4-8 and 5-8 to get the higher genus invariants. It follows from Mumford's formula

$$
\left(1+c_{1}\left(E^{\vee}\right)+c_{2}\left(E^{\vee}\right)+\cdots+c_{g}\left(E^{\vee}\right)\right) \cdot\left(1+c_{1}(E)+c_{2}(E)+\cdots+c_{g}(E)\right)=1
$$

that

$$
\begin{aligned}
\left(\tilde{t}^{g}+c_{1}\left(E^{\vee}\right) \tilde{t}^{g-1}+c_{2}\left(E^{\vee}\right) \tilde{t}^{g-2}+\cdots+c_{g}\left(E^{\vee}\right)\right) . \\
\\
\left(\tilde{t}^{g}+c_{1}(E) \tilde{t}^{g-1}+c_{2}(E) \tilde{t}^{g-2}+\cdots+c_{g}(E)\right)=\tilde{t}^{2 g} .
\end{aligned}
$$

Hence we have

$$
\begin{aligned}
{\left[\int_{\left[\mathbf{M}_{g, \mu}^{\mathrm{rel}}\left(\mathbf{P}^{\mathrm{1}}\right)_{0}\right]^{\text {vir }}} c_{\text {top }}(V)\right]_{\Theta_{I}} } & =\left[\frac{1}{\left|\mathrm{~A}_{\Theta_{I}}\right|} \int_{\bar{M}_{g, 1}} \frac{d \cdot \tilde{t}^{2 g-1}}{\tilde{t}-d \cdot \psi} c_{g}(E)\right]_{0} \\
& =d^{2 g-2} \int_{\bar{M}_{g, 1}} \psi^{2 g-2} c_{g}(E),
\end{aligned}
$$

where in the last equality we have used the fact that the moduli space $\bar{M}_{g, 1}$ of DeligneMumford stable curves has dimension $\operatorname{dim}_{\mathbb{C}} \bar{M}_{g, 1}=3 g-2$. The Hodge integral above can be easily evaluated by using Faber and Pandharipande's generating function for Hodge integrals over the moduli space $\bar{M}_{g, 1}$ [2]. Taking the result from [2], we conclude that

$$
\left[\int_{\left[\mathbf{M}_{g, \mu}^{\text {rel }}\left(\mathbf{P}^{1}\right)_{0}\right]^{\text {vir }}} c_{\text {top }}(V)\right]_{\Theta_{I}}=d^{2 g-2} \frac{2^{2 g-1}-1}{2^{2 g-1}} \frac{\left|B_{2 g}\right|}{(2 g) !} .
$$

As promised, 5-11 is precisely equal to the expected answer 1-3. 


\section{Invariants for $h>1$}

As mentioned before, all invariants for $h>1$ vanish. The main idea that underlies our argument is that at least one of the weights of the $S^{1}$-action on the obstruction bundle is zero. We will present our argument for genus-zero and higher genus cases separately.

We will first consider the genus-zero case. Assume that $h=2$, in which case $\mu=\left(d_{1}, d_{2}\right)$, where $d_{1}+d_{2}=d$. In genus-zero $C=C_{1} \sqcup_{p} C_{2}$, where $C_{1}$ and $C_{2}$ both are rational curves and $p$ is a node that gets mapped to $q_{0}$. For $i=1$ or $2, d_{i}>0$ is the degree of the map $f_{i}$ that maps $C_{i}$ to $\mathbf{P}^{1}$. If we denote the pre-images of $q_{\infty}$ by $x_{1} \in C_{1}$ and $x_{2} \in C_{2}$, then we have the normalization exact sequence

$$
\begin{aligned}
0 \longrightarrow \mathcal{O}_{C}\left(-d_{1} x_{1}-d_{2} x_{2}\right) & \longrightarrow \mathcal{O}_{C_{1}}\left(-d_{1} x_{1}\right) \oplus \mathcal{O}_{C_{2}}\left(-d_{2} x_{2}\right) \\
\left.\mathcal{O}_{C}\left(-d_{1} x_{1}-d_{2} x_{2}\right)\right|_{p} & \longrightarrow 0,
\end{aligned}
$$

which gives the long exact sequence of cohomology

$$
\begin{aligned}
0 \longrightarrow \mathrm{H}^{0}\left(C,\left.\mathcal{O}_{C}\left(-d_{1} x_{1}-d_{2} x_{2}\right)\right|_{p}\right) & \longrightarrow \mathrm{H}^{1}\left(C, \mathcal{O}_{C}\left(-d_{1} x_{1}-d_{2} x_{2}\right)\right) \longrightarrow \\
\longrightarrow \mathrm{H}^{1}\left(C_{1}, \mathcal{O}_{C_{1}}\left(-d_{1} x_{1}\right)\right) \oplus \mathrm{H}^{1}\left(C_{2}, \mathcal{O}_{C_{2}}\left(-d_{2} x_{2}\right)\right) & \longrightarrow 0
\end{aligned}
$$

From this we immediately see that one of the $S^{1}$-action weights on $\mathrm{H}^{1}\left(C, \mathcal{O}_{C}\left(-d_{1} x_{1}-\right.\right.$ $\left.\left.d_{2} x_{2}\right)\right)$ is zero, since the weight on $\mathrm{H}^{0}\left(C,\left.\mathcal{O}_{C}\left(-d_{1} x_{1}-d_{2} x_{2}\right)\right|_{p}\right)$ is zero. This means that the contribution of $\mathrm{H}^{1}\left(C, \mathcal{O}_{C}\left(-d_{1} x_{1}-d_{2} x_{2}\right)\right)$ to the equivariant top Chern class of the obstruction bundle vanishes, thus rendering the invariant to vanish as well.

For $h=3, C$ contains a contracted genus-zero component $\tilde{C}_{0}$ which is connected to 3 rational curves, say $C_{1}, C_{2}, C_{3}$, at 3 nodes, say $p_{1}, p_{2}, p_{3}$. Note that since $\tilde{C}_{0}$ contains 3 special points, it is stable and can be contracted to $q_{0}$. Each $C_{i}$ maps to $\mathbf{P}^{1}$ with degree $d_{i}>0$ and contains a special point $x_{i}$ that gets mapped to $q_{\infty}$. As usual there is the exact normalization sequence

$$
\left.0 \longrightarrow \mathcal{O}_{C}\left(-\sum_{i}^{3} d_{i} x_{i}\right) \longrightarrow \bigoplus_{i=1}^{3} \mathcal{O}_{C_{i}}\left(-d_{i} x_{i}\right) \oplus \mathcal{O}_{\tilde{C}_{0}} \longrightarrow \bigoplus_{i=1}^{3} \mathcal{O}_{C}\left(-\sum_{i}^{3} d_{i} x_{i}\right)\right|_{p_{i}} \longrightarrow 0
$$

and the associated cohomology long exact sequence

$$
\begin{array}{r}
0 \longrightarrow \mathrm{H}^{0}\left(\tilde{C}_{0}, \mathcal{O}_{\tilde{C}_{0}}\right) \\
\stackrel{\psi}{\longrightarrow} \bigoplus_{i}^{3} \mathrm{H}^{0}\left(C,\left.\mathcal{O}_{C}\left(-\sum_{i}^{3} d_{i} x_{i}\right)\right|_{p_{i}}\right) \longrightarrow \mathrm{H}^{1}\left(C, \mathcal{O}_{C}\left(-\sum_{i}^{3} d_{i} x_{i}\right) \longrightarrow\right. \\
\longrightarrow \bigoplus_{i=1}^{3} \mathrm{H}^{1}\left(C_{i}, \mathcal{O}_{C_{i}}\left(-d_{i} x_{i}\right)\right) \oplus \mathrm{H}^{1}\left(\tilde{C}_{0}, \mathcal{O}_{\tilde{C}_{0}}\right) \longrightarrow 0 .
\end{array}
$$


Here, $\psi$ is not surjective and it follows that not all of the zero weights on

$$
\bigoplus_{i}^{3} \mathrm{H}^{0}\left(C,\left.\mathcal{O}_{C}\left(-\sum_{i}^{3} d_{i} x_{i}\right)\right|_{p_{i}}\right)
$$

get cancelled in

$$
\begin{gathered}
\mathrm{H}^{1}\left(C, \mathcal{O}_{C}\left(-\sum_{i}^{3} d_{i} x_{i}\right)\right)=\bigoplus_{i=1}^{3} \mathrm{H}^{1}\left(C_{i}, \mathcal{O}_{C_{i}}\left(-d_{i} x_{i}\right) \oplus \mathrm{H}^{1}\left(\tilde{C}_{0}, \mathcal{O}_{\tilde{C}_{0}}\right)-\mathrm{H}^{0}\left(\tilde{C}_{0}, \mathcal{O}_{\tilde{C}_{0}}\right)\right. \\
+\bigoplus_{i}^{3} \mathrm{H}^{0}\left(C,\left.\mathcal{O}_{C}\left(-\sum_{i}^{3} d_{i} x_{i}\right)\right|_{p_{i}}\right) .
\end{gathered}
$$

Therefore, the equivariant top Chern class of the obstruction bundle in the localization theorem vanishes. We can perform induction on $h$ and conclude that genus-zero invariants vanish for all $h>1$. We will now sketch how that works. For $h \leq n$, assume that the $S^{1}$-action on $\mathrm{H}^{1}\left(C, \mathcal{O}_{C}\left(-\sum_{i}^{h} d_{i} x_{i}\right)\right)$ has at least one zero weight and that therefore the invariants vanish. At $h=n+1$, a $S^{1}$-fixed stable map can be constructed from that at $h=n$ by attaching a rational curve $C_{n+1}$ to the contracted component, such that $\operatorname{deg}\left(\left.f\right|_{C_{n+1}}\right)=d_{n+1}>0 . C_{n+1}$ contains the point $x_{n+1}$ that gets mapped to $q_{\infty}$ and is joined to the contracted component at a new node. Such an operation increases the number of nodes by 1 , and analyzing the exact normalization sequence and its associated cohomology long exact sequence shows that the number of zero weights on $\mathrm{H}^{1}\left(C, \mathcal{O}_{C}\left(-\sum_{i}^{h} d_{i} x_{i}\right)\right)$ has increased by one. Therefore, the total number of zero weights on $\mathrm{H}^{1}\left(C, \mathcal{O}_{C}\left(-\sum_{i}^{h} d_{i} x_{i}\right)\right)$ is again non-zero. This shows that the equivariant top Chern class of the obstruction bundle vanishes at $h=n+1$.

Now assume that $g \geq 1$ and $h=2$. In addition to the two rational curves $C_{1}$ and $C_{2}$, we introduce a stable genus- $g$ curve $\tilde{C}_{g}$, which gets contracted to $q_{0}$. There are two nodes $p_{1}$ and $p_{2}$ where $C_{1}$ and $C_{2}$, respectively, intersect $\tilde{C}_{g}$. In our usual notation, the normalization exact sequence in the present case is

$$
\begin{aligned}
0 \longrightarrow \mathcal{O}_{C}\left(-d_{1} x_{1}-d_{2} x_{2}\right) \longrightarrow & \bigoplus_{i=1}^{2} \mathcal{O}_{C_{i}}\left(-d_{i} x_{i}\right) \oplus \mathcal{O}_{\tilde{C}_{g}} \longrightarrow \\
\left.\bigoplus_{i=1}^{2} \mathcal{O}_{C}\left(-d_{1} x_{1}-d_{2} x_{2}\right)\right|_{p_{i}} & \longrightarrow 0 .
\end{aligned}
$$

This implies the following long exact sequence of cohomology:

$$
\begin{aligned}
0 & \longrightarrow \mathrm{H}^{0}\left(\tilde{C}_{g}, \mathcal{O}_{\tilde{C}_{g}}\right) \\
& \stackrel{\varphi}{\longrightarrow} \mathrm{H}^{0}\left(C,\left.\mathcal{O}_{C}\left(-d_{1} x_{1}-d_{2} x_{2}\right)\right|_{p_{1}}\right) \oplus \mathrm{H}^{0}\left(C,\left.\mathcal{O}_{C}\left(-d_{2} x_{2}-d_{2} x_{2}\right)\right|_{p_{2}}\right) \longrightarrow \\
& \mathrm{H}^{1}\left(C, \mathcal{O}_{C}\left(-d_{1} x_{1}-d_{2} x_{2}\right)\right) \longrightarrow \bigoplus_{i=1}^{2} \mathrm{H}^{1}\left(C_{i}, \mathcal{O}_{C_{i}}\left(-d_{i} x_{i}\right)\right) \oplus \mathrm{H}^{1}\left(\tilde{C}_{g}, \mathcal{O}_{\tilde{C}_{g}}\right) \longrightarrow 0 .
\end{aligned}
$$


Unlike in the $h=1$ case, $\varphi$ is not surjective and we need to compute

$$
\begin{aligned}
\mathrm{H}^{1}\left(C, \mathcal{O}_{C}\right. & \left.\left(-d_{1} x_{1}-d_{2} x_{2}\right)\right) \\
& =\bigoplus_{i=1}^{2} \mathrm{H}^{1}\left(C_{i}, \mathcal{O}_{C_{i}}\left(-d_{i} x_{i}\right)\right) \oplus \mathrm{H}^{1}\left(\tilde{C}_{g}, \mathcal{O}_{\tilde{C}_{g}}\right)-\mathrm{H}^{0}\left(\tilde{C}_{g}, \mathcal{O}_{\tilde{C}_{g}}\right)+ \\
& +\mathrm{H}^{0}\left(C,\left.\mathcal{O}_{C}\left(-d_{1} x_{1}-d_{2} x_{2}\right)\right|_{p_{1}}\right) \oplus \mathrm{H}^{0}\left(C,\left.\mathcal{O}_{C}\left(-d_{2} x_{2}-d_{2} x_{2}\right)\right|_{p_{2}}\right) .
\end{aligned}
$$

The zero weight term from $\mathrm{H}^{0}\left(\tilde{C}_{g}, \mathcal{O}_{\tilde{C}_{g}}\right)$ will cancel only one of the two zero weight terms from the second line, leaving a zero weight term in $\mathrm{H}^{1}\left(C, \mathcal{O}_{C}\left(-d_{1} x_{1}-d_{2} x_{2}\right)\right)$. Hence the equivariant top Chern class of the obstruction bundle again vanishes, and so does the invariant.

The vanishing of the invariants for $g \geq 1$ again follows from induction on $h$. As in the genus-zero case, a $S^{1}$-fixed stable map at $h=n+1$ can be constructed from that at $h=n$ by attaching a non-contracted rational curve, say $C_{n+1}$, to the contracted component $\tilde{C}_{g}$ at a new node. This addition of a node increases the number of zero weights of the $S^{1}$-action on $\mathrm{H}^{1}\left(C, \mathcal{O}_{C}\left(-\sum_{i}^{h} d_{i} x_{i}\right)\right)$, and therefore the equivariant top Chern class of the obstruction bundle vanishes at $h=n+1$ as it does at $h=n$. Hence, all higher genus invariants vanish for $h>1$.

To recapitulate, we have just established that

$$
\left[\int_{\left[\mathbf{M}_{g, \mu}^{\mathrm{rel}}\left(\mathbf{P}^{1}\right)\right]^{\mathrm{vir}}} c_{\mathrm{top}}(V)\right]_{\Theta_{I}}=0, \quad \forall g \geq 0, d \geq h>1,
$$

in perfect agreement with what was expected from Section 1.

\section{Conclusion}

In this paper we have made an explicit connection between topological open string theory and relative stable morphisms. In the particular example we consider, we have successfully reproduced open string instanton multiple cover answers as invariants of relative stable maps. So far several interesting proposals for studying open string instanton effects have been made (Ooguri-Vafa [16], Kachru-Katz-Lawrence-McGreevy [8] and Aganagic-Vafa [1]), but direct computational methods involving integrals over moduli spaces of stable morphisms have been hitherto lacking. This is in marked contrast to the closed string case, where there exist well-developed techniques in the context of Gromov-Witten theory (Faber, Graber and Pandharipande [5, 2]). 
Open string instantons play an important role in string theory. For example, in the Strominger-Yau-Zaslow conjecture of mirror symmetry, open string instanton effects are crucial for modifying the geometry of D-brane moduli space [20]. Also, genus-zero topological open string amplitudes are important for computing superpotentials in $\mathcal{N}=1$ supersymmetric theories in 4 -dimension-see $[8,16]$ and references therein. It is clear that many illuminating implications can stem from understanding better how one can directly compute open string instanton amplitudes. We hope we have made it clear in this paper that relative stable morphisms could be the right framework for studying open string instantons in general, and that the proposed link between topological open string theory and relative stable maps well deserve further investigations.

Applying the theory of relative stable morphisms to topological open string theory is in the incipient stage. In a sense we have studied in this paper what could be considered the simplest example. As mentioned in Section 1, the quantities we have reproduced correspond to the invariants of a simple knot in $S^{3}$. Labastida, Marino and Vafaof [10] have extended the results in [16] to more non-trivial knots and links, and have described how to construct Lagrangian submanifolds, for torus links at least, on the topological string theory side of the duality. It will be interesting to apply our method to those cases as well. Also, Aganagic and Vafa have recently announced some interesting results on counting holomorphic discs in Calabi-Yau 3-folds [1], and we would like to understand their results by means of relative stable morphisms.

Acknowledgments The second author gratefully acknowledges initial collaboration on this project and numerous useful discussions with Jun S Song. He also thanks Kefeng Liu for discussions and correspondence.

The first author's research is supported in part by an NSF grant, a Sloan fellowship and a Terman fellowship. The second author's research is supported in part by an NSF Graduate Fellowship and the US Department of Energy under contract DE-AC03-76SF00515.

\section{References}

[1] M Aganagic, C Vafa, Mirror symmetry, D-branes and counting holomorphic discs (2000) arXiv:hep-th/0012041

[2] C Faber, R Pandharipande, Hodge integrals and Gromov-Witten theory, Invent. Math. 139 (2000) 173-199 MR1728879 
[3] K Fukaya, K Ono, Arnold conjecture and Gromov-Witten invariant for general symplectic manifolds, from: "The Arnoldfest (Toronto, ON, 1997)", Fields Inst. Commun. 24, Amer. Math. Soc., Providence, RI (1999) 173-190 MR1733575

[4] R Gopakumar, C Vafa, On the gauge theory/geometry correspondence, Adv. Theor. Math. Phys. 3 (1999) 1415-1443 MR1796682

[5] T Graber, R Pandharipande, Localization of virtual classes, Invent. Math. 135 (1999) 487-518 MR1666787

[6] E-N Ionel, T H Parker, Gromov-Witten invariants of symplectic sums, Math. Res. Lett. 5 (1998) 563-576 MR1664598

[7] E-N Ionel, T H Parker, Relative Gromov-Witten invariants, Ann. of Math. (2) 157 (2003) 45-96 MR1954264

[8] S Kachru, S Katz, A Lawrence, J McGreevy, Mirror symmetry for open strings, Phys. Rev. D (3) 62 (2000) 126005, 14 MR1814377

[9] S Katz, C-C M Liu, Enumerative geometry of stable maps with Lagrangian boundary conditions and multiple covers of the disc, from: "The interaction of finite-type and Gromov-Witten invariants at the Banff International Research Station (2003)", Geom. Topol. Monogr. 8 (2006) 119-142

[10] J M F Labastida, M Mariño, C Vafa, Knots, links and branes at large N, J. High Energy Phys. (2000) Paper 7, 42 MR1806596

[11] A-M Li, Y Ruan, Symplectic surgery and Gromov-Witten invariants of Calabi-Yau 3-folds, Invent. Math. 145 (2001) 151-218 MR1839289

[12] J Li, Relative Gromov-Witten invariants and a degeneration formula of Gromov-Witten invariants, in preparation

[13] J Li, A degeneration of stable morphisms and relative stable morphisms (2000) arXiv: math.AG/0009097

[14] J Li, G Tian, Virtual moduli cycles and Gromov-Witten invariants of general symplectic manifolds, from: "Topics in symplectic 4-manifolds (Irvine, CA, 1996)", First Int. Press Lect. Ser., I, Internat. Press, Cambridge, MA (1998) 47-83 MR1635695

[15] J Li, G Tian, Virtual moduli cycles and Gromov-Witten invariants of algebraic varieties, J. Amer. Math. Soc. 11 (1998) 119-174 MR1467172

[16] H Ooguri, C Vafa, Knot invariants and topological strings, Nuclear Phys. B 577 (2000) 419-438 MR1765411

[17] Y Ruan, Topological sigma model and Donaldson-type invariants in Gromov theory, Duke Math. J. 83 (1996) 461-500 MR1390655

[18] Y Ruan, G Tian, A mathematical theory of quantum cohomology, J. Differential Geom. 42 (1995) 259-367 MR1366548 
[19] B Siebert, Symplectic Gromov-Witten invariants, from: "New trends in algebraic geometry (Warwick, 1996)", London Math. Soc. Lecture Note Ser. 264, Cambridge Univ. Press, Cambridge (1999) 375-424 MR1714832

[20] A Strominger, S-T Yau, E Zaslow, Mirror symmetry is T-duality, Nuclear Phys. B 479 (1996) 243-259 MR1429831

[21] E Witten, Topological sigma models, Comm. Math. Phys. 118 (1988) 411-449 MR958805

[22] E Witten, Quantum field theory and the Jones polynomial, Comm. Math. Phys. 121 (1989) 351-399 MR990772

Department of Mathematics, Stanford University

Stanford, California 94305, USA

Department of Physics, and SLAC, Stanford University

Stanford, California 94305, USA

jli@math.stanford.edu, yss@leland.stanford.edu

Received: 26 April 2001 